\section{COMENTARIOS A LOS ARTÍCULOS: "PREVALENCIA ANUAL DE TRASTORNOS Y USO DE SERVICIOS DE SALUD MENTAL EN EL PERÚ: RESULTADOS DEL ESTUDIO MUNDIAL DE SALUD MENTAL, 2005" Y "PREVALENCIA DE VIDA Y EDAD DE INICIO DE TRASTORNOS MENTALES EN EL PERÚ URBANO: RESULTADOS DEL ESTUDIO MUNDIAL DE SALUD MENTAL, 2005"}

\begin{abstract}
COMMENTS TO THE ARTICLES "ANNUAL PREVALENCE OF MENTAL DISORDERS AND USE OF MENTAL HEALTH SERVICES IN PERU: RESULTS OF THE WORLD MENTAL HEALTH STUDY, 2005" AND "LIFETIME PREVALENCE AND AGE OF ONSET OF MENTAL DISORDERS IN PERU: RESULTS OF THE WORLD MENTAL HEALTH STUDY, 2005 “
\end{abstract}

\section{Javier E. Saavedra $1,2, a$}

Sr. Editor. Los estudios de carga de morbilidad realizados por el Ministerio de Salud (MINSA) han demostrado que los trastornos mentales constituyen uno de los principales problemas, inclusive entre otras patologías físicas ${ }^{(1)}$. La Encuesta Mundial de Salud Mental (EMSM) auspiciada por la OMS representó una iniciativa importante para propiciar que los estados asuman una función más efectiva para enfrentar este problema. En el 2004 la Dirección General de Promoción de la Salud del MINSA, tuvo a bien asignar el liderazgo del equipo técnico de la Encuesta al Instituto Nacional de Salud Mental "Honorio Delgado - Hideyo Noguchi" (INSM“HDHN"), con la participación y financiamiento del Instituto Nacional de Salud (INS) y los auspicios administrativos de la OPS, correspondiendo la operación de campo al Instituto Nacional de Estadística e Informática (INEI).

La adaptación del proyecto matriz a la realidad peruana fue liderado por el equipo de investigadores del INSM“HD-HN" *. Estas actividades incluyeron: la

\footnotetext{
Instituto Nacional de Salud Mental Honorio Delgado-Hideyo Noguchi. Lima, Perú.

Universidad Peruana Cayetano Heredia. Lima, Perú.

a Médico psiquiatra

Recibido: 17-10-14 Aprobado: 05-11-14
}

Citar como: Saavedra JE. Comentarios a los artículos: "Prevalencia anual de trastornos y uso de servicios de salud mental en el Perú: resultados del estudio mundial de salud mental, 2005" y "Prevalencia de vida y edad de inicio de trastornos mentales en el Perú urbano: resultados del estudio mundial de salud mental, 2005” [carta]. Rev Peru Med Exp Salud Publica. 2014;31(4):806-7. "peruanización" del instrumento (CIDI) y la elaboración y/o revisión de diversos materiales (cuaderno de respuestas, atlas psicofarmacológico, glosario, manual del entrevistador, plan de control de calidad y plan de capacitación de la encuesta piloto). El protocolo de investigación fue remitido para aprobación por el Comité de Investigación del INS y el Comité de Ética el 27 mayo de 2004, con el nombre de "Encuesta nacional de hogares sobre salud y estilos de vida (ENHSEV)", siendo el primer suscrito el investigador principal, acompañado por profesionales del INSM"HD-HN" y del INS. Asimismo, el equipo técnico participó y colaboró en la capacitación y selección de los encuestadores, en la supervisión directa de la encuesta piloto, así como en el acompañamiento y supervisión de la operación de campo ejecutada por el INEI. La data obtenida sería analizada en México y, posteriormente, remitida de regreso para la redacción del artículo por el equipo responsable. Durante los siguientes años de culminada la operación de campo no recibimos comunicación alguna, y en los últimos meses hemos tomado conocimiento de la aparición de dos artículos ${ }^{(2,3)}$, realizados con la base de datos de este proyecto. En dicho artículo se hace se agradece a los "funcionarios del MINSA y del INSM"HD-HN" sin tomar en consideración el grado de participación del equipo en el desarrollo de la investigación, y afirmándose, además, que los autores de la investigación son los autores del artículo.

En cuanto a las limitaciones del estudio convendría precisar que no solo no se incluyeron las ciudades más pobres, sino tampoco aquellas con mayores problemas psicosociales, a diferencia de la cobertura nacional en otros países. También debemos señalar que el instrumento original fue construido y validado principalmente en países desarrollados, por lo que su desempeño en otras partes del mundo podría no ser culturalmente satisfactoria, según se desprende de la amplia variabilidad de resultados en los distintos países, como lo menciona el propio reporte del Consorcio de la Encuesta Mundial de Salud Mental (The WHO World Mental Health Survey Consortium) ${ }^{(4,5)}$. Otras limitaciones que requieren mencionarse en el referido proyecto tienen que ver con la duración de las entrevistas y con el tiempo trascurrido desde su realización, hace 9 años, durante los cuales se han producido grandes cambios socioeconómicos en el país.

La iniciativa de la Encuesta Mundial auspiciada por la OMS representa una gestión encomiable para colocar la salud mental en el foco de atención de las políticas en los diferentes países y representó un esfuerzo notable de los investigadores del INSM "HD-HN". Consideramos que tal empresa merece el reconocimiento respectivo. Juzgamos oportuno que futuras iniciativas internacionales deben tomar en cuenta las variaciones 
socioculturales y psicológicas de los países al diseñar los instrumentos de evaluación, de otra manera podríamos caer en una paradoja metodológica donde el empleo del mismo instrumento no hace comparable los resultados.

Fuentes de financiamiento: autofinanciado.

Conflictos de interés: los autores declaran no tener conflictos de interés.

Investigadores del INSM"HD-HN" que participaron en el proyecto: Santos Gregorio González Farfán, Luis Bernardo Guimas Reyna, Julio Raúl Huamán Pineda, María de los Ángeles Mendoza Vilca, Ysela Marlene Nicolás Hoyos, Martín Nizama Valladolid, Yolanda Isabel Robles Arana, Vilma Zoila Paz Schaeffer, Rolando Marcial Pomalima Rodríguez, Abe Sagástegui Soto, Santiago Martín Stucchi Portocarrero, Carmen Violeta Vargas Palomino

\section{REFERENCIAS BIBLIOGRÁFICAS}

1. Velásquez A. Estimación de la carga de enfermedad en el Perú. Promoviendo alianzas y estrategias. Lima: Abt Associates Inc; 2006. p. 50.

2. Fiestas F, Piazza M. Prevalencia de vida y edad de inicio de trastornos mentales en el Perú urbano: Resultados del estudio mundial de salud mental, 2005. Rev Peru Med Exp Salud Publica. 2014;31(1):39-47.

3. Piazza M, Fiestas F. Prevalencia anual de trastornos y uso de servicios de salud mental en el Perú: resultados del estudio mundial de salud mental, 2005. Rev Peru Med Exp Salud Publica. 2014;31(1):30-8.

4. Demyttenaere K, Bruffaerts R, Posada-Villa J, Gasquet I, Kovess V, Lepine JP, et al. Prevalence severity, and unmeet need for treatment of mental disorders in the World Health Organization World Mental Health Surveys. JAMA. 2004 Jun 2; 291(21):2581-90.

5. Rosenman S. Cause for caution: culture, sensitivity and the World Mental Health Survey Initiative. Australasian Psychiatry. 2012 Feb;20(1):14-9. doi: 10.1177/1039856211430149.

Correspondencia: Javier Esteban Saavedra Castillo

Dirección: Jr. Eloy Espinoza Saldaña 709. San Martín de Porres, Perú.

Teléfono: 6149200 Anexo 1057

Correo electrónico: saavedra@terra.com.pe
COMENTARIOS A LOS ARTÍCULOS: "PREVALENCIA ANUAL DE TRASTORNOS Y USO DE SERVICIOS DE SALUD MENTAL EN EL PERÚ: RESULTADOS DEL ESTUDIO MUNDIAL DE SALUD MENTAL, 2005" Y "PREVALENCIA DE VIDA Y EDAD DE INICIO DE TRASTORNOS MENTALES EN EL PERÚ URBANO: RESULTADOS DEL ESTUDIO MUNDIAL DE SALUD MENTAL, 2005" - RÉPLICA DE LOS AUTORES

\author{
COMMENTS TO THE ARTICLES: "ANNUAL \\ PREVALENCE OF MENTAL DISORDERS AND USE \\ OF MENTAL HEALTH SERVICES IN PERU: RESULTS \\ OF THE WORLD MENTAL HEALTH STUDY, 2005" \\ AND "LIFETIME PREVALENCE AND AGE OF ONSET \\ OF MENTAL DISORDERS IN PERU: RESULTS OF \\ THE WORLD MENTAL HEALTH STUDY, 2005" - \\ AUTHORS REPLY
}

$$
\text { Marina Piazza } a^{1,2, a} \text {, Fabián Fiestas }{ }^{1, b}
$$

Sr. Editor. El estudio de Salud Mental Mundial en el Perú es una investigación promovida por la Organización Mundial de la Salud (OMS-OPS) y la Universidad de Harvard. Esta última firmó un acuerdo con el MINSA comprometiéndose a brindar apoyo en recojo de información, codificación de diagnósticos, certificación de base de datos, análisis de datos y preparación de publicaciones. EI INS, la OPS y el INEI, establecieron acuerdos, donde el INS asume el financiamiento del estudio, la OPS la coordinación técnica y la DGSP la supervisión. La OPS firmó un convenio con el INEI para implementar la encuesta y entregar la base de datos y el informe técnico.

El equipo del MINSA, con participación de profesionales del INSM, sugirió cambios al instrumento, y apoyó al INEI en el 2004 en el piloto y capacitación de entrevistadores, tal como se describe en el Informe Final del INEI ${ }^{(1)}$. EI INEI

\footnotetext{
Instituto Nacional de Salud. Lima, Perú

Universidad Peruana Cayetano Heredia. Lima, Perú

psicóloga epidemióloga; ${ }^{\mathrm{b}}$ médico epidemiólogo

Recibido: 11-11-14 Aprobado: 19-11-14

Citar como: Piazza M, Fiestas F. Comentarios a los artículos: "Prevalencia anual de trastornos y uso de servicios de salud mental en el Perú: resultados del estudio mundial de salud mental, 2005" y "Prevalencia de vida y edad de inicio de trastornos mentales en el Perú urbano: resultados del estudio mundial de salud mental, 2005” - Réplica de los autores [carta]. Rev Peru Med Exp Salud Publica. 2014;31(4):807-8.
} 